\title{
Editorial
}

\section{Dark Energy and Modified Gravity}

Dark energy problem is considered now as one of the main challenges for XXI century physics. It is quite possible that modified gravity may suggest the gravitational solution for above problem of late-time acceleration of the universe. Simultaneously, modified gravity may describe also the early inflationary universe, thus unifying two accelerating epochs and describing the whole sequence of the universe evolution eras: inflation, radiation/matter dominance and dark energy.

Different modified gravities: $F(R), F(G)$, string-inspired scalar-Gauss-Bonnet gravity, non-minimally coupled modified gravities etc. are proposed as models for such early-time and/or late-time accelerating expansion.

The current hot topic is devoted to review and to discussion of new results in this approach which gives the gravitational alternative for accelerating expansion, i.e. inflation or dark energy.

The idea is very attractive due to fact that according to it, there is no need in the introduction of extra dark components: dark energy, inflaton and/or dark matter. Apart from modified gravity, several works of this issue are devoted to the discussion of dark energy and/or inflation in frames of fluid/scalar theory models.

\section{S. Nojiri}

Department of Physics

Nagoya University

Nagoya 464-8602

Japan

nojiri@phys.nagoya-u.ac.jp
S.D. Odintsov

Consejo Superior de Investigaciones Cientficas (ICE/CSIC) and Institut d'Estudis Espacials de Catalunya (IEEC)

Campus UAB, Facultat Ciències

Torre C5-Par-2a pl

E-08193 Bellaterra (Barcelona)

Spain

odintsov@aliga.ieec.uab.es

(C) Odintsov and Nojiri; Licensee Bentham Open.

This is an open access article licensed under the terms of the Creative Commons Attribution Non-Commercial License (http://creativecommons.org/ licenses/by-nc/3.0/) which permits unrestricted, non-commercial use, distribution and reproduction in any medium, provided the work is properly cited. 